\title{
Real time quantification of low temperature radiofrequency ablation lesion size using phased array intracardiac echocardiography in the canine model: comparison of two dimensional images with pathological lesion characteristics
}

\author{
A Doi, M Takagi, I Toda, M Teragaki, M Yoshiyama, K Takeuchi, J Yoshikawa
}

See end of article for authors' affiliations

Correspondence to: Dr Masahiko Takagi, Department of Internal Medicine and Cardiology,

Osaka City University

Graduate School of

Medicine, Abeno-ku,

Japan; m7424580@

msic.med.osaka-cu.ac.jp

Accepted

19 December 2002

\begin{abstract}
Objective: To evaluate the feasibility of quantifying low temperature radiofrequency catheter ablation (RFCA) lesions using a phased array intracardiac echocardiography (ICE) catheter - with better tissue penetration and in a deflectable device-in the canine model.

Intervention: Low temperature radiofrequency (RF) energy $\left(50-60^{\circ} \mathrm{C}\right.$ at up to $\left.40 \mathrm{~W}\right)$ was delivered to the left ventricle in 11 beagles for 60 seconds, using an 8 French catheter with a deflectable tip and a $4 \mathrm{~mm}$ distal electrode.

Main outcome measures: Comparison of the width and depth of RFCA lesions measured by ICE with pathological findings.

Results: 33 RF energies were delivered in 11 dogs. 31 lesions (94\%) were confirmed at necropsy. 27 of 31 ablation lesions (87\%) were detected by ICE. The mean (SD) width and depth of the ICE detected lesions were $10.4(2.6) \mathrm{mm}$ and 5.7 (1.9) mm, respectively. Pathological findings showed that RFCA lesions consisted of inner and outer layers. Macroscopically, the mean (SD) width and depth of the inner layers were $7.6(2.3) \mathrm{mm}$ and $3.6(1.2) \mathrm{mm}$ and those for the whole layers were $10.0(2.8) \mathrm{mm}$ and $5.3(1.5) \mathrm{mm}$, respectively. Microscopically, the inner and outer layers corresponded to necrotic and oedematous areas, respectively. The ICE detected lesion size had better correlation with the pathological measurements of the whole layers in width $(r=0.911)$ and in depth $(r=0.756)$.

Conclusion: The real time evaluation of RFCA lesion size using the phased array ICE is feasible, even with a low temperature RF application. However, ICE slightly overestimates RFCA lesion size compared with pathological necrotic lesion size.
\end{abstract}

$\mathrm{R}$ adiofrequency catheter ablation (RFCA) has been established as one of the treatment options for many cardiac tachyarrhythmias and the success rates in most reported series are very high.$^{1-4}$ However, in some patients, applications of radiofrequency (RF) energy fail to achieve the desired electrophysiological effect because of adequate but mistargeted lesion creation or ablation lesions of inadequate volume.

Intracardiac echocardiography (ICE) has been firmly established as an important diagnostic tool for imaging cardiac structures, assessing cardiac function, and diagnosing cardiovascular abnormalities..$^{5-8}$ ICE also provides image monitoring during cardiac electrophysiological procedures, which may be used to guide interatrial septal puncture or to position catheters for mapping or ablation..$^{9-13}$ Recently, a new phased array ICE catheter, with better tissue penetration and in a deflectable device, has become available. ${ }^{14-18}$ However, quantification and characterisation of the images of the low temperature RFCA lesions in the clinical setting, using the phased array ICE, are still insufficient.

This study was designed to evaluate the feasibility of quantifying low temperature RFCA lesions using phased array ICE in vivo and to compare the ICE detected lesions with the pathological findings in the canine model.

\section{METHODS}

\section{Animal preparation}

Eleven adult beagles of each sex (weight $8-12 \mathrm{~kg}$ ) were anaesthetised with intravenously administered sodium pentobarbital ( $25 \mathrm{mg} / \mathrm{kg})$, intubated, and continuously ventilated. A
10 French and an 8 French sheath introducer (Terumo Co, Tokyo, Japan) were inserted into the femoral vein and artery, respectively. The analogue blood pressure waveforms and surface ECG were monitored throughout the procedure. All animal handling and experimental procedures, including death, were conducted in a gentle manner to minimise stress and discomfort to the dogs. Each animal received humane care in compliance with the principles of laboratory animal care of the National Society of Medical Research and National Academy of Sciences.

\section{ICE catheters}

A new deflectable 10 French phased array ICE catheter (AcuNav, Acuson, Mountain View, California, USA) with an $8.5 \mathrm{MHz}$ imaging frequency was used in this study. The catheter is $90 \mathrm{~cm}$ long and has a 64 element phased array ultrasound transducer. The catheter tip can be deflected $160^{\circ}$ in two planes (anterior to posterior and right to left) and locked in position. This probe produces long axis images with tissue penetration to a depth of $15 \mathrm{~cm}$. The ICE catheter was introduced through a 10 French sheath in the femoral vein in to the right ventricle under fluoroscopy and ultrasound navigation. Long axis images of the left ventricle were obtained.

Abbreviations: ICE, intracardiac echocardiography; RF, radiofrequency; RFCA, radiofrequency catheter ablation 


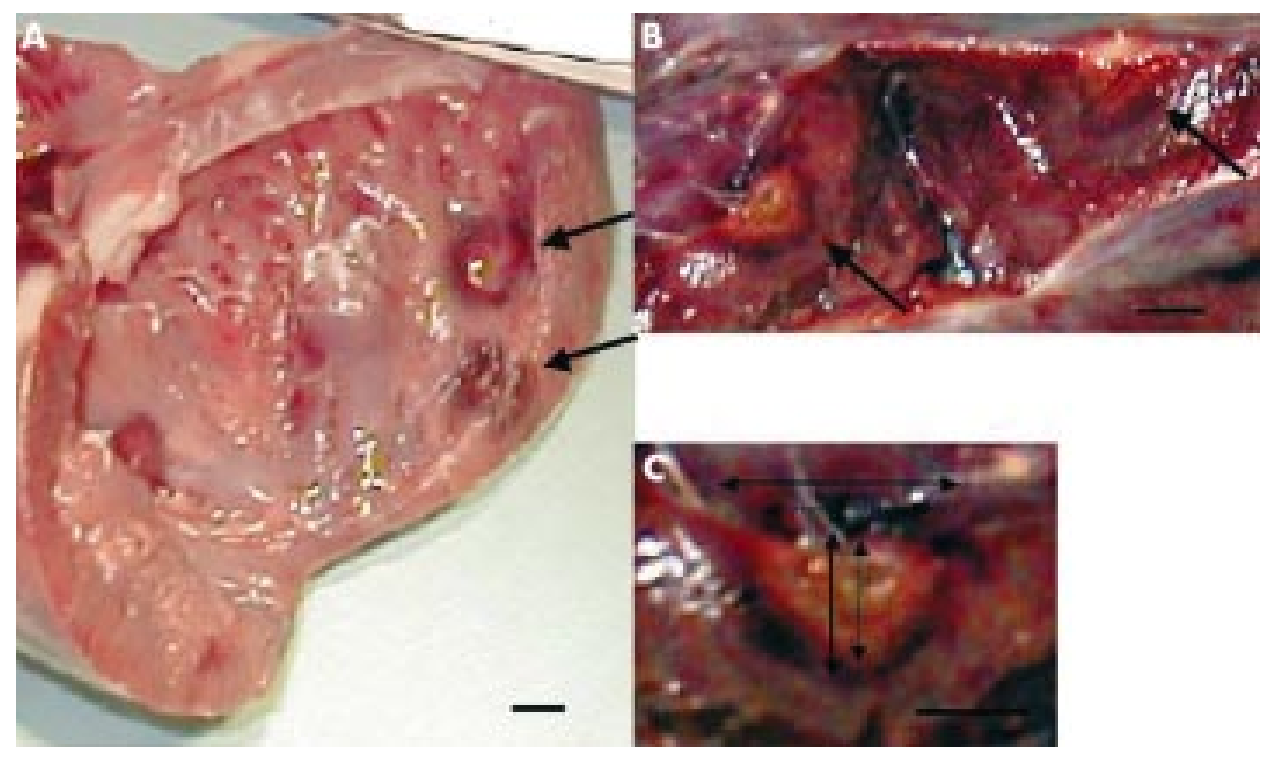

Figure 1 Representative pathological findings of radiofrequency catheter ablation (RFCA) lesions. (A) Findings on the endocardial surface. (B) Findings sectioned parallel to the long axis of the lesion in a plain perpendicular to the endocardial surface. (C)

Magnified image of RFCA lesion in panel (B). Macroscopic findings showed that RFCA lesions (arrows in panels (A) and (B)) consisted of two discrete layers (inner and outer). Dotted arrows and unbroken arrows in panel $(C)$ indicate the width and depth of the inner layer and of the whole lesion (including inner and outer layers), respectively. Bars $=5$ $\mathrm{mm}$.

\section{RFCA}

An 8 French ablation catheter with a deflectable tip and a $4 \mathrm{~mm}$ distal electrode (EP Technologies Inc, San Jose, California, USA) was introduced into the left ventricle through the femoral artery under fluoroscopy and ultrasound navigation. RF energy was applied only when good catheter-tissue contact with the left ventricular wall was confirmed by ICE as previously reported, ${ }^{12}$ and the amplitudes of unipolar and bipolar ventricular electrograms were assessed. Once good contact was confirmed, the catheter was not repositioned until energy application was completed. RFCA was performed with unmodulated RF current from a temperature controlled generator (EP Technologies Inc). For each ablation, intracardiac electrograms, current, power, temperature, and impedance were continuously recorded from the distal electrode tip. RF energy was continuously delivered to obtain a catheter tip temperature of $50-60^{\circ} \mathrm{C}$ at up to $40 \mathrm{~W}$ for 60 seconds, which was the same as that used in the clinical setting. Each lesion was attempted at three points (base, midline, and apex) in the posterior wall of the left ventricle in each animal. We chose three lesions that were far enough apart and sufficiently distinct that we could distinguish them by using fluoroscopic and ICE imaging. We waited five minutes to measure the width and depth of the RFCA lesions at end systole with standard two dimensional images by ICE because the ICE detected lesion size had not changed visually after three minutes. Lesion width was defined as the longest axis. Lesion depth was measured from the endocardial surface to the deepest point in the lesion.

\section{Pathological evaluation}

Just after lesion size was measured by ICE, potassium chloride was fully infused under deep anaesthesia, the animals were killed, and the heart was immediately excised. Macroscopically, the RFCA lesions consisted of two layers (inner and outer). We measured the width and depth of the inner layer and of the whole layers including inner and outer layers (fig 1). Each of the lesions was blocked and was sectioned parallel to the long axis of the lesion in a plane perpendicular to the endocardial surface. After fixation in formalin, the sections were stained with haematoxylin and eosin for microscopic examination of the lesions.

\section{Statistical analysis}

Data are presented as mean (SD). Correlation between ICE and pathology for lesion width and depth was tested by linear regression analysis. A paired $t$ test was performed to assess the significance of bias between ICE and pathology, and to identify the standard deviation of the differences. Differences were considered significant at a value of $\mathrm{p}<0.05$. The measurement of lesion width and depth was assessed for interobserver and intraobserver variability. Intraobserver variability was determined from duplicate measurements. Interobserver variability was determined from measurements by two cardiologists who were blinded to data information. These observer variabilities were assessed according to the Bland-Altman method.

\section{RESULTS}

\section{Visualisation of ablation catheter}

With ICE imaging, the ablation catheter tip was visualised and catheter-tissue contact was easily evaluated (fig 2). Neither microbubble formation nor coagulum was observed in any case. During RFCA, there were no procedural complications.

\section{Identification of RF ablation lesions}

Thirty three RF energies were delivered in 11 dogs. Every RF application resulted in adequate tissue temperature $\left(>50^{\circ} \mathrm{C}\right)$ for all lesions. The mean temperature achieved during all RF applications was $58.7(7.1)^{\circ} \mathrm{C}$. The mean power required to achieve these temperatures was 23.3 (12.7) W. The RFCA lesions were identified by ICE as well circumscribed hyperechoic areas and were clearly distinguished from normal myocardium (fig 2). Thirty one lesions (94\%) were confirmed

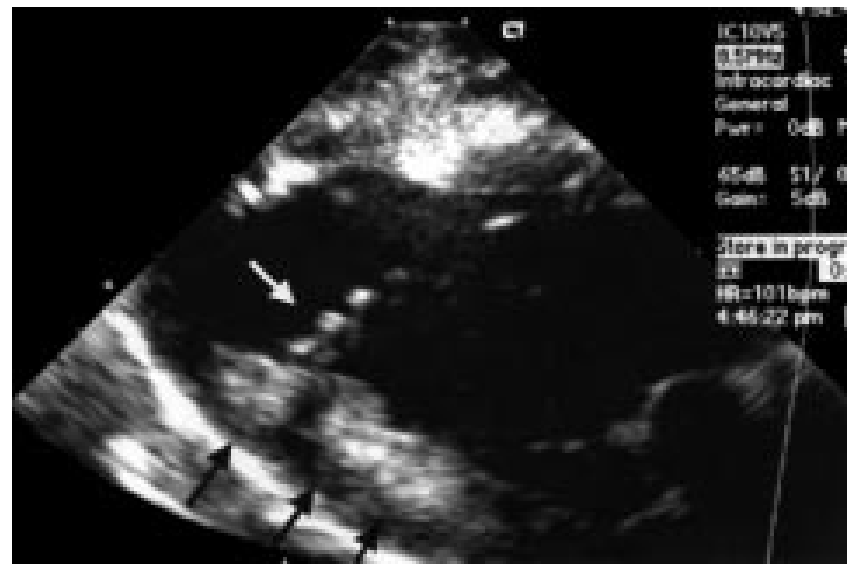

Figure 2 A representative intracardiac echocardiography (ICE) image of RFCA lesions at end systole. The ablation catheter was easily visualised (white arrow). The three RFCA lesions were identified as well circumscribed hyperechoic areas in the posterior wall of the left ventricle (black arrows). 
Table 1 Width and depth of radiofrequency catheter ablation lesions measure by intracardiac echocardiography (ICE) and pathologically

\begin{tabular}{llll}
\hline & & \multicolumn{2}{l}{ Pathology } \\
\cline { 3 - 4 } & ICE & Inner layer & $\begin{array}{l}\text { Inner plus } \\
\text { outer layer }\end{array}$ \\
\hline Lesion width $(\mathrm{mm})$ & $10.4(2.6)$ & $7.6(2.3)$ & $10.0(2.8)$ \\
Lesion depth $(\mathrm{mm})$ & $5.7(1.9)$ & $3.6(1.2)$ & $5.3(1.5)$ \\
\hline
\end{tabular}

Data are mean $(S D)$.

at necropsy, of which 27 lesions (87\%) were detected by ICE. The difference in detection rate between the three sites (base, midline, and apical posterior wall) was not significant.

\section{Measurements of RFCA lesions}

We evaluated the 27 lesions detected by ICE. The mean width and depth of the lesions imaged by ICE were 10.4 (2.6) $\mathrm{mm}$ and 5.7 (1.9) $\mathrm{mm}$, respectively (table 1). Pathological findings showed that ablation lesions consisted of two layers: inner and outer (fig 1). Macroscopically, the mean width and depth of the inner layers were $7.6(2.3) \mathrm{mm}$ and $3.6(1.2) \mathrm{mm}$, and those of the whole ablation lesions-the sum of inner and outer layers-were $10.0(2.8) \mathrm{mm}$ and 5.3 (1.5) $\mathrm{mm}$, respectively. There were good correlations between the ICE detected lesion size and the pathological measurements of inner layer in width (fig 3A, $r=0.696, p=0.004$ ) and in depth (fig 4A, $r=0.616, \mathrm{p}=0.017)$. However, there was better correlation between the ICE detected lesion size and the pathological measurements of the whole ablation lesions in width (fig 3B, $r=0.911, \mathrm{p}<0.0001$ ) and in depth (fig 4B, $r=0.756$, $\mathrm{p}<0.0002)$. Microscopically, the inner and outer layers corresponded to a necrotic and degenerative area and an oedematous area with bleeding, respectively (fig 5). There were no transmural lesions and no lesion had thrombus on the endocardial surface.

\section{Observer variability}

The interobserver and intraobserver variabilities for the width and depth from random lesions were excellent. There were good correlations for the width $(r=0.994, \mathrm{p}<0.001$ and $r=0.999, \mathrm{p}<0.001)$ and for the depth $(r=0.991, \mathrm{p}<0.0001$ and $r=0.992, \mathrm{p}<0.0001)$.

\section{DISCUSSION}

The findings of the present study indicate that the phased array ICE catheter can identify a substantial number of RFCA lesions in real time, even using low temperature RF application as in the clinical setting. The ICE detected lesion size had better correlation with lesions that consisted of necrosis, degeneration, and oedema of myocardium. These findings also showed that ICE slightly overestimated the true pathological necrotic area.
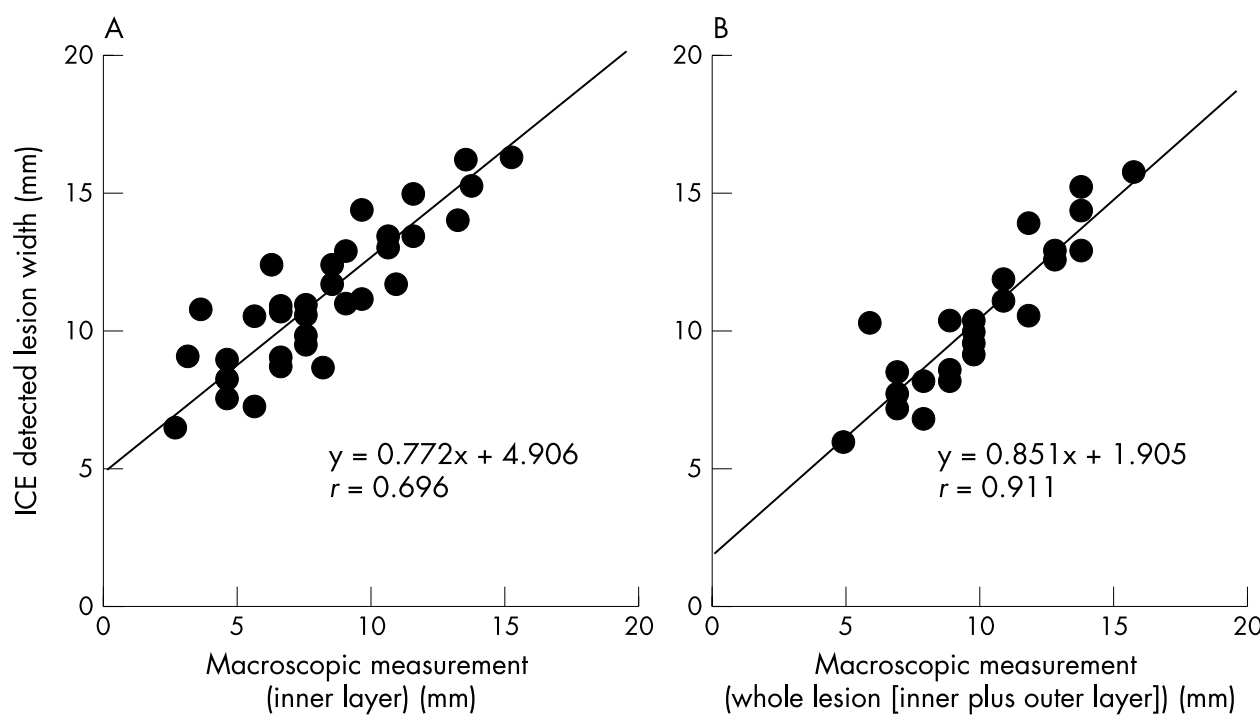

Figure 3 Correlation between ICE detected lesion size and pathological measurements for lesion width. The ICE detected lesion width has better correlation with macroscopic measurements of the whole RFCA lesions $(B)$ than with those of the inner layers (A).
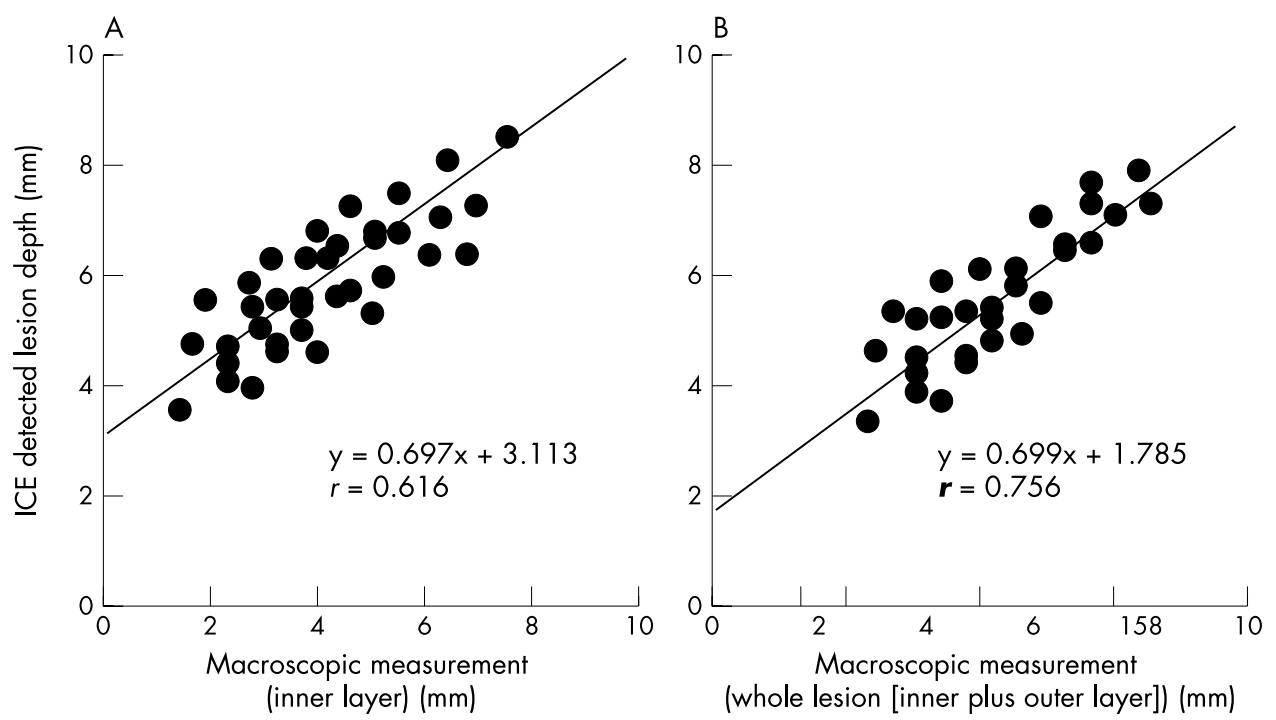

Figure 4 Correlation between ICE detected lesion size and pathological measurements for lesion depth. The ICE detected lesion depth has better correlation with macroscopic measurements of the whole RFCA lesions (B) than with those of the inner layers (A).

(whole lesion [inner plus outer layer]) (mm) 


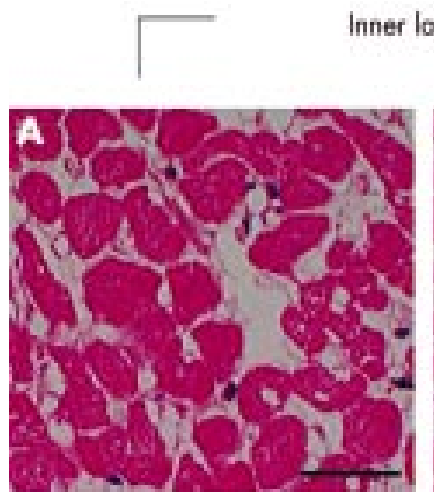

Inner loyer

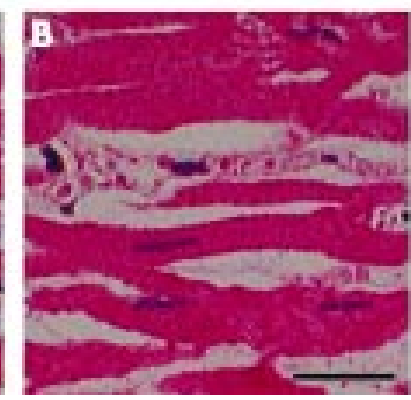

Outer loyer

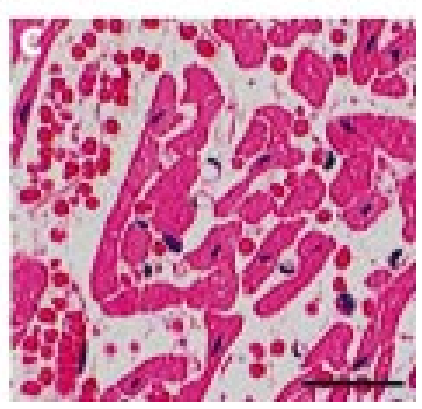

Figure 5 Representative microscopic findings of RFCA lesions. Haematoxylin and eosin stain. (A) Necrosis of myocardium. (B) Degeneration of myocardium. (C) Interstitial and myocardial oedema with bleeding. The inner and outer layers corresponded to a necrotic and degenerative area and an oedematous area with bleeding, respectively. Bars $=20 \mu \mathrm{m}$.
Previous studies also indicated the feasibility of using ICE to visualise intracardiac structures, guide catheterisation, and assess catheter-tissue contact..$^{5-13}$ Some of these studies assessed RFCA lesions using ICE..$^{8}{ }^{11-13}$ Most of these studies, however, used ICE catheters with a high frequency (that is, more than $9 \mathrm{MHz}$ ) transducer rotated by an external motor. As the penetration of these catheters was limited, the quantification of RFCA lesions was restricted to superficial lesions in the neighbourhood of the ICE catheter. ${ }^{19-21}$

Recently, a new phased array ICE catheter with better tissue penetration, in a deflectable device, has become available. Some investigators examined RFCA lesions detected by the phased array ICE. However, in these reports, high temperature and high power RF application or an irrigation tipped catheter was used. ${ }^{15-17}$ In clinical studies, we usually use lower temperature and lower power RF application for safety. In the present study, we quantified RFCA lesions by phased array ICE using low temperature RF application as in the clinical setting. To the best of our knowledge, this is the first study to evaluate the feasibility of phased array ICE using low temperature RF application and to compare the two dimensional images with pathological lesion characteristics in vivo. Previous studies reported microbubble formation and coagulum in the myocardium and that transmural lesions were created in some cases. ${ }^{13}{ }^{19-21}$ However, in this study, none of these were observed. We suggest that these phenomena were associated with high power and high temperature RF application and that these findings are unusual in the clinical setting.

\section{Clinical implications}

For RFCA of ventricular tachycardia with structural heart diseases, atrial flutter, atrial fibrillation, and re-entrant atrial tachycardia complicating reparative surgery for congenital heart diseases, assessment of ablation lesion volume may gain in importance in the successful creation of continuous and long, linear lesions. ${ }^{22}{ }^{23}$ In the present study, we found that ICE actually overestimated pathological necrotic areas. The difference in the width between ICE detected lesions and pathological necrotic areas was 3.17 (1.93) mm (26.6 (15.5)\%) and the difference in the depth was 2.03 (1.16) $\mathrm{mm}(35.7$ (17.2)\%). Recognising these differences we suggest that, to create linear lesions precisely, RF energies should be applied consecutively, overlapping the lesions imaged by ICE. To distinguish necrotic areas from oedematous areas in real time a new technology, such as contrast agents, would be needed. Recently, the utility of the phased array ICE in human studies has been shown. ${ }^{15-17}$ Although no studies have been published regarding assessment of RFCA lesions in humans using the phased array ICE, the new ICE should be a useful tool for confirming RFCA lesions more precisely in clinical studies.

\section{Study limitations}

In the present study, we measured the width and depth of the RFCA lesions after a five minute delay because the ICE detected lesion size did not exhibit further visual change after three minutes. Moreover, among the three RFCA lesions in each dog, there was a difference in time between completion of measurement by ICE and pathological measurement. As all lesions were treated in the same way, however, it is unlikely that the delay would have altered the conclusions.

Two lesions $(6 \%)$ were not found on pathological examination. Previous reports indicated a similar proportion of missing lesions in studies of RFCA in the ventricle. ${ }^{11}{ }^{12}{ }^{19-21}$

\section{Conclusions}

The real time evaluation of RFCA lesion size using the phased array ICE is feasible in vivo, even with a low temperature RF application. However, the ICE detected area has better correlation with the whole lesion, including degenerative and oedematous areas of the myocardium. Actually, ICE slightly overestimates pathological necrotic areas.

\section{Authors' affiliations}

A Doi, M Takagi, I Toda, M Teragaki, M Yoshiyama, K Takeuchi,

J Yoshikawa, Department of Internal Medicine and Cardiology, Osaka City University Graduate School of Medicine, Osaka, Japan

\section{REFERENCES}

1 Jackman WM, Wang XZ, Friday KJ, et al. Catheter ablation of accessory atrioventricular pathways (Wolff- Parkinson-White syndrome) by radiofrequency current. N EnglJ Med 1991;324:1605-11.

2 Lesh MD, Van Hare GF, Schamp DJ, et al. Curative percutaneous catheter ablation using radiofrequency energy for accessory pathways in all locations: results in 100 consecutive patients. J Am Coll Cardiol 1992;19:1303-9.

3 Calkins H, Sousa J, el-Atassi R, et al. Diagnosis and cure of the Wolff-Parkinson-White syndrome or paroxysmal supraventricular tachycardias during a single electrophysiologic test. N Engl J Med $1991 ; 324: 1612-8$

4 Nath S, DiMarco JP, Haines DE. Basic aspects of radiofrequency catheter ablation. J Cardiovasc Electrophysiol 1994;5:863-76.

5 Ren JF, Schwartzman D, Callans D, et al. Imaging technique and clinical utility for electrophysiologic procedures of lower frequency $(9 \mathrm{MHz})$ intracardiac echocardiography. Am J Cardiol 1998;82:1557-60.

6 Schwartz SL, Gillam LD, Weintraub AR, et al. Intracardiac echocardiography in humans using a small-sized (6F), low frequency (12.5 MHz) ultrasound catheter. Methods, imaging planes and clinical experience. J Am Coll Cardiol 1993;21:189-98.

7 Olgin JE, Kalman JM, Fitzpatrick AP, et al. Role of right atrial endocardial structures as barriers to conduction during human type I atrial flutter. Activation and entrainment mapping guided by intracardiac echocardiography. Circulation 1995;92:1839-48.

8 Kalman JM, Olgin JE, Karch MR, et al. Use of intracardiac echocardiography in interventional electrophysiology. Pacing Clin Electrophysiol 1997;20:2248-62.

9 Chu E, Kalman JM, Kwasman MA, et al. Intracardiac echocardiography during radiofrequency catheter ablation of cardiac arrhythmias in humans. J Am Coll Cardiol 1994;24:1351-7.

10 Mitchel JF, Gillam LD, Sanzobrino BW, et al. Intracardiac ultrasound imaging during transseptal catheterization. Chest 1995;108:104-8.

11 Olgin JE, Kalman JM, Chin M, et al. Electrophysiological effects of long, linear atrial lesions placed under intracardiac ultrasound guidance. Circulation 1997;96:2715-21.

12 Roithinger FX, Steiner PR, Goseki Y, et al. Low-power radiofrequency application and intracardiac echocardiography for creation of continuous left atrial linear lesions. J Cardiovasc Electrophysiol 1999;10:680-91. 
13 Epstein LM, Mitchell MA, Smith TW, et al. Comparative study of fluoroscopy and intracardiac echocardiographic guidance for the creation of linear atrial lesions. Circulation 1998;98:1796-801.

14 Bruce CJ, Packer DL, Seward JB. Intracardiac Doppler hemodynamics and flow: new vector, phased-array ultrasound-tipped catheter. Am J Cardiol 1999;83:1509-12

15 Bruce CJ, Packer DL, Seward JB. Transvascular Imaging: feasibility study using a vector phased array ultrasound catheter. Echocardiography 1999; 16:425-30.

16 Morton JB, Sanders P, Kalman JM, et al. Phased-array intracardiac echocardiography to guide radiofrequency ablation in the left atrium and at the pulmonary vein ostium. J Cardiovasc Electrophysiol 2001;12:343-8.

17 Packer DL, Stevens CL, Curley MG et al. Intracardiac phased-array imaging: methods and initial clinical experience with high resolution, under blood visualization: initial experience with intracardiac phased-array ultrasound. J Am Coll Cardiol 2002;39:509-16.
18 Teragaki $M$, Toda I, Takagi $M$, et al. New applications of intracardiac echocardiography: assessment of coronary blood flow by colour and pulsed Doppler imaging in dogs. Heart 2002;88:283-8.

19 Kalman JM, Jue J, Sudhir K, et al. In vitro quantification of radiofrequency ablation lesion size using intracardiac echocardiography in dogs. Am J Cardiol 1996:77:217-9.

20 Chugh SS, Chan RC, Packer DL, et al. Catheter tip orientation affects radiofrequency ablation lesion size in the canine left ventricle. Pacing Clin Electrophysiol 1999;22:413-20.

21 Chan RC, Seward JB, Packer DL, et al. The effect of ablation electrode length and catheter tip to endocardial orientation on radiofrequency lesion size in the canine right atrium. Pacing Clin Electrophysiol 2002;25:4-13.

22 Haissaguerre $M$, Shah DC, Jais $P$, et al. Electrophysiological breakthroughs from the left atrium to the pulmonary veins. Circulation 2000;102:2463-5

23 Pappone C, Rosaino E, Oreto G, et al. Circumferential radiofrequency ablation of pulmonary vein ostia. Circulation 2000;102:2619-28.

\section{IMAGES IN CARDIOLOGY}

\section{Apical thrombus associated with left ventricular apical ballooning}

A 64 year old woman under antidepressant treatment, but with no coronary risk factors, was admitted to our institution with a two day history of continuous atypical chest pain. The ECG showed pronounced ST segment elevation in leads II, III, aVF and V2-V6 (panel A). Coronary angiography revealed normal coronary arteries and left ventriculography demonstrated apical asynergy with basal hyperkinesia ("apical ballooning"). A striking filling defect-highly suggestive of a thrombus - was also viewed at the apex (panel B, diastole; panel C, systole). Left ventricular ejection fraction (LVEF) was $40 \%$. The apical ballooning, intraventricular thrombus, and a dynamic intraventricular pressure gradient of $70 \mathrm{~mm} \mathrm{Hg}$ were confirmed by transthoracic echocardiography (TTE). Maximal creatine phosphokinase and troponin I values were $159 \mathrm{U} / \mathrm{l}$ (normal $0-170 \mathrm{U} / \mathrm{l}$ ) and $7.4 \mathrm{ng} / \mathrm{ml}$ (normal 0-0.1 ng/ml), respectively. Nevertheless, pathologic Q waves developed from V1 to V3. The patient was discharged under anticoagulant treatment. Three months later the ECG showed no Q waves but just negative $\mathrm{T}$ waves on V3-V6 (panel D). TTE showed absolutely normal left ventricular wall motion, an LVEF of $62 \%$, and complete resolution of the apical thrombus and intraventricular pressure gradient. Anticoagulation was discontinued.

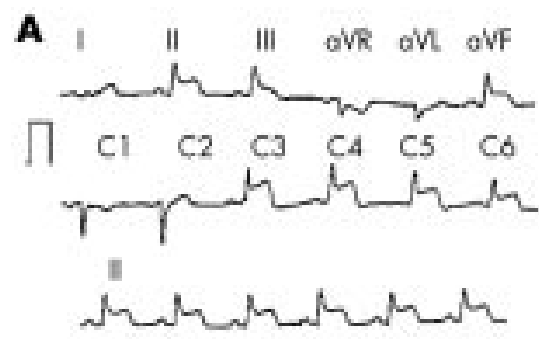

D

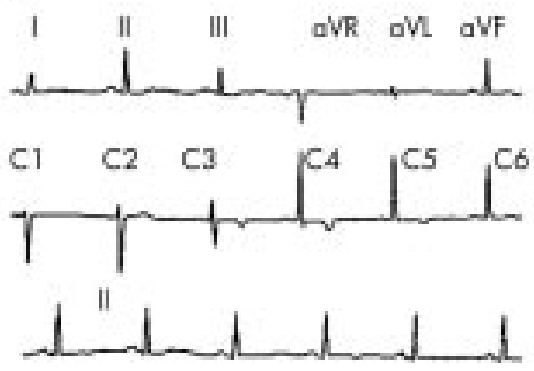

Apical ballooning has been recently described. Clinical presentation includes chest pain, ST segment elevation on ECG, and minimal or no rise of cardiac enzymes in patients with normal angiographic coronary arteries, but with a peculiarly shaped left ventricle. Full recovery of left ventricular wall motion should occur within weeks. Although some major complications have been

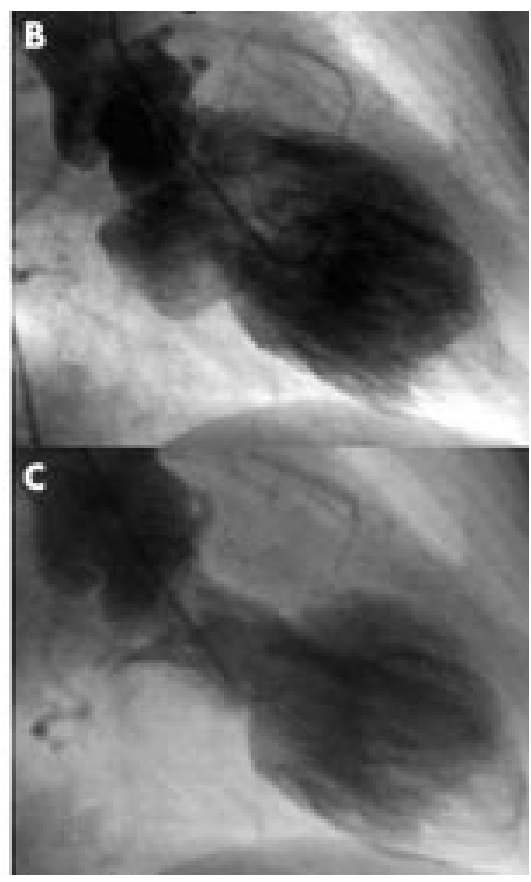

associated with this entity (namely, heart failure and arrhythmias) the clinical course and prognosis is favourable in most cases. Apical thrombus has not been previously described in patients with apical ballooning.

C F Barrera-Ramirez J M Jimenez-Mazuecos F Alfonso jimenezmazuecos@hotmail.com 